\title{
Temperature Dependence of the Stability of Ion Pair Interactions, and its Implications on the Thermostability of Proteins from Thermophiles
}

\author{
SWETHA BIKKINA, AGASTYA P BHATI, SILADITYA PADHI and U DEVA PRIYAKUMAR* \\ Center for Computational Natural Sciences and Bioinformatics, International Institute of Information \\ Technology, Hyderabad, Telengana 500 032, India \\ Email: deva@iiit.ac.in
}

MS received 9 November 2016; revised 4 January 2017; accepted 7 January 2017

\begin{abstract}
An understanding of the determinants of the thermal stability of thermostable proteins is expected to enable design of enzymes that can be employed in industrial biocatalytic processes carried out at high temperatures. A major factor that has been proposed to stabilize thermostable proteins is the high occurrence of salt bridges. The current study employs free energy calculations to elucidate the thermodynamics of the formation of salt bridge interactions and the temperature dependence, using acetate and methylguanidium ions as model systems. Three different orientations of the methylguanidinium approaching the carboxylate group have been considered for obtaining the free energy profiles. The association of the two ions becomes more favorable with an increase in temperature. The desolvation penalty corresponding to the association of the ion pair is the lowest at high temperatures. The occurrence of bridging water molecules between the ions ensures that the ions are not fully desolvated, and this could provide an explanation for the existence of internal water molecules in thermostable proteins reported recently. The findings provide a detailed picture of the interactions that make ion pair association at high temperatures a favorable process, and reaffirm the importance of salt bridges in the design of thermostable proteins.
\end{abstract}

Keywords. Thermostable proteins; ion pairs; salt bridges; molecular dynamics; free energy calculations

\section{Introduction}

Thermophilic and hyperthermophilic organisms are microorganisms that survive at high temperatures, and have adopted strategies for thriving at such temperatures. ${ }^{1-5}$ While hyperthermophiles display optimum growth at temperatures higher than $100^{\circ} \mathrm{C}$, thermophiles can survive between 50 and $100^{\circ} \mathrm{C}$. Mesophiles, on the other hand, are organisms that can survive at normal conditions below $45^{\circ} \mathrm{C}$. The machinery that enables (hyper)thermophiles to exist at such high temperatures is the presence of thermostable proteins. ${ }^{4,5}$ These proteins perform their function optimally at these temperatures without being denatured. Proteins from such organisms usually show extreme thermal stability, despite having folded structures very similar to their mesophilic counterparts. ${ }^{4,5}$ The structural features that render this thermostability to thermophilic proteins have drawn a lot of interest, since incorporation of these features into mesophilic proteins can be a strategy for the design of thermostable proteins. ${ }^{6-8}$ The thermal stability of these proteins is appealing because of its potential applications in

*For correspondence industrial bio-catalysis, whereby thermophilic enzymeassisted reactions can be carried out at high temperatures, possibly achieving high yield and faster rate. ${ }^{9-11}$ Furthermore, these proteins have been of great interest because they can serve as model systems for obtaining insights into the factors that could stabilize a protein. ${ }^{2,3}$

Several studies have attempted to identify the determinants that impart thermal stability to proteins from thermophiles. While a few studies have suggested that hydrophobic interactions could stabilize these proteins, ${ }^{12,13}$ other studies have shown that salt bridges (interactions between oppositely charged residues) render this stability to thermostable proteins. ${ }^{14-16}$ In fact, networks of ion pairs have been reported to exist on the surface as well as the interface between subunits in these proteins. ${ }^{15,16}$ Molecular dynamics studies on hyperthermophilic proteins have also shown that a combination of ion pair and hydrophobic interactions can stabilize the protein. ${ }^{6,17-20}$ The flexibility of the protein could also contribute to thermostability, with hyperthermophilic proteins being more rigid as a result of stronger intramolecular interactions. ${ }^{21,22}$ Another factor that plays a role in thermostability is the solvent, and both surface water molecules and internal water 
molecules are known to be crucial. ${ }^{23-25}$ However, at high temperatures, thermostable proteins lose some of these coordinating water molecules, implying that interactions between residues are more direct. ${ }^{18,23}$

The effect of ion pair and hydrophobic interactions on the stability of a protein has been investigated by employing small model systems that resemble the naturally occurring amino acids. ${ }^{26-36}$ These model systems can accurately capture electrostatic/hydrophobic interactions between amino acids while at the same time reducing the computational complexity. Molecular simulation studies on freely diffusing amino acids show that, while the number of hydrophobic contacts decreases with an increase in temperature, salt bridge contacts are less sensitive to changes in temperature. ${ }^{28}$

Thus, although a number of studies have suggested that electrostatic interactions between oppositely charged residues are the major determinant of thermostability, it would be noteworthy to quantitatively investigate at an atomistic detailed level the factors that stabilize electrostatic interactions at high temperatures, since such an explanation is lacking. This study uses methylguanidium and acetate as model systems for positively and negatively charged residues, respectively, and provides detailed insights into the nature and strength of interactions between them at different temperatures by employing molecular dynamics-based free energy calculations. Stabilization of ion pairs at high temperatures is attributed to lower desolvation penalty accompanying the association of ion pairs. The orientation of the oppositely charged groups relative to each other and the occurrence of bridging water molecules are shown to be critical factors in determining stability. Based on the findings, possible roles for conserved salt bridges and internal water molecules in thermostable proteins are discussed.

\section{Computational}

All calculations were performed using the CHARMM program with the all-atom CHARMM22 protein force field with CMAP corrections and the TIP3P water model. ${ }^{37-40}$ A cubic water box of $40 \AA \times 40 \AA \times 40 \AA$ containing 2054 water molecules was built centered at the midpoint of the constrained ion pair. A cutoff of $18 \AA$ was used for the nonbonded interactions, including essentially all interactions in the cluster. Periodic boundary conditions were set up using CRYSTAL module in CHARMM, ${ }^{37}$ and the particle mesh Ewald method was used for treating long range electrostatics. ${ }^{41}$ In all cases, the atoms were constrained to move on a straight line and the side chains were constrained to remain in specific orientations. These constraints were implemented using the MMFP module in CHARMM. All calculations were performed in the presence of the SHAKE algorithm to constrain covalent bonds containing hydrogen atoms. ${ }^{42}$ Umbrella sampling calculations were performed on one or more well-defined orientations of acetate and methylguanidium, using the distance between the nearest heavy atoms as the reaction coordinate. A total of three different orientations were examined, as shown in Figure 1, and details about the three orientations and the restraining potential applied in each of these orientations are described below.

Orientation I. C1 and N3 of methylguanidium and C1 and $\mathrm{C} 2$ of acetate were constrained on the same line, and $\mathrm{N} 1, \mathrm{~N} 2$, and $\mathrm{C} 2$ of methylguanidium and $\mathrm{O} 1$ and $\mathrm{O} 2$ of acetate were constrained on the same plane. The reaction coordinate $r$ is the distance between $\mathrm{N} 1$ of methylguanidium and $\mathrm{O} 1$ of acetate.

Orientation II. N2 and C1 of methylguanidium and C1 and $\mathrm{C} 2$ of acetate were constrained on the same line and $\mathrm{N} 3, \mathrm{~N} 2$, and $\mathrm{C} 2$ of methylguanidium and $\mathrm{O} 1$ and $\mathrm{O} 2$ of acetate were constrained in the same plane. The reaction coordinate $r$ is the distance between $\mathrm{N} 3$ of methylguanidium and $\mathrm{O} 1$ of acetate.

Orientation III. N1 and C1 of methylguanidium and $\mathrm{C} 1$ and $\mathrm{C} 2$ of acetate were constrained on the same

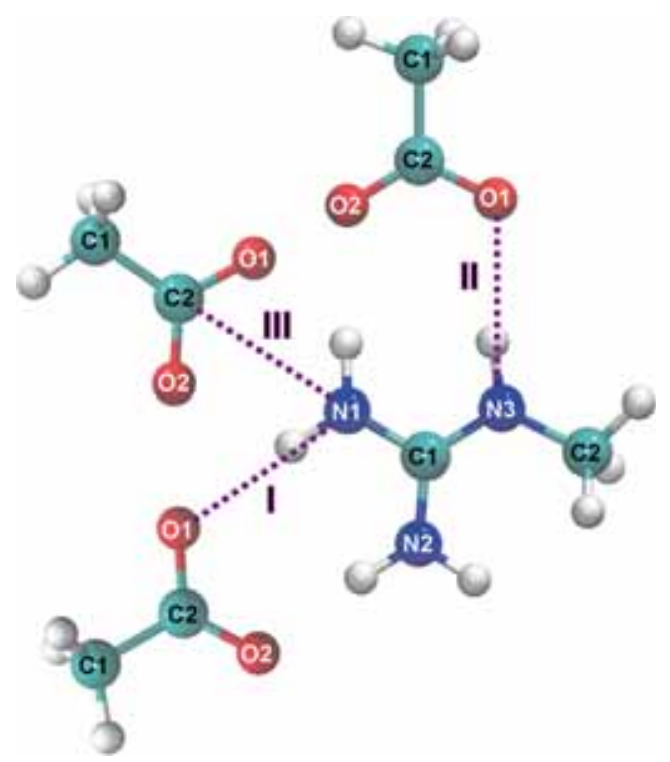

Figure 1. Restraints applied for the three different orientations of acetate relative to methylguanidium ion. The different carbon, oxygen, and nitrogen atoms are named $\mathrm{C} 1$, $\mathrm{C} 2, \mathrm{O} 1, \mathrm{O} 2$, and so on, for the sake of convenience. In orientation I, the restraint is on the distance between N1 of methylguanidium and $\mathrm{O} 1$ of acetate. In orientation II, it is on the distance between $\mathrm{N} 3$ of methylguanidium and $\mathrm{O} 1$ of acetate, and in orientation III, on the distance between N1 of methylguanidium and $\mathrm{C} 2$ of acetate. 
line and $\mathrm{N} 3, \mathrm{~N} 2$, and $\mathrm{C} 2$ of methylguanidium and $\mathrm{O} 1$ and $\mathrm{O} 2$ of acetate were constrained in the same plane. The reaction coordinate $r$ is the distance between $\mathrm{N} 1$ of methylguanidium and $\mathrm{C} 2$ of acetate.

For applying a potential at desired positions along the reaction coordinate, a harmonic potential of the form,

$$
w_{i}(r)=k_{i}\left(r-r_{0}\right)^{2}
$$

was used, where $k_{i}$ is the force constant for window $i, r_{0}$ is the center of the window $i$ along the reaction coordinate, and $r$ is the instantaneous position along the reaction coordinate. The weighted histogram analysis method (WHAM) ${ }^{43,44}$ was used to obtain the unbiased free energy from the biased distribution of $r$ using the relation

$$
A_{i}(r)=-k_{B} T \ln P_{i}(r)-w_{i}(r)+F_{i}
$$

where $k_{B}$ is the Boltzmann constant, $T$ is the temperature, $P_{i}(r)$ is the biased distribution, and $F_{i}$ is a constant for window $i$.

A total of 7 windows were used for each orientation of methylguanidium/acetate, with the distance varying from $3 \AA$ to $9 \AA$ at intervals of $1 \AA$. A harmonic potential with force constant $k=2 \mathrm{kcal} \mathrm{mol}^{-1} \AA^{-2}$ was employed in order to restrain the distance between the desired atoms. In some cases, this force constant was found insufficient to sample near the top of high energy barriers, and additional simulations were performed using a harmonic potential with force constant $k=14 \mathrm{kcal} \mathrm{mol}^{-1} \AA^{-2}$ centered at the barrier.

For all the three orientations, the umbrella sampling calculations were performed at five independent temperatures $(300,350,400,450$, and $500 \mathrm{~K})$. For each window, the system was minimized and then equilibrated for $20 \mathrm{ps}$ in the presence of positional restraints on the solute of force constant $5.0 \mathrm{kcal} \mathrm{mol}^{-1} \AA^{-2}$. This was followed by production runs for $3 \mathrm{~ns}$ in the NPT ensemble with a time-step of 2 fs. The analysis of trajectories was performed on the last $1 \mathrm{~ns}$ of the simulations, considering the initial part as equilibration period. The snapshots of the structures depicted in this paper were made using the VMD program, ${ }^{45}$ and trajectory analyses were performed using the CHARMM program. ${ }^{36}$
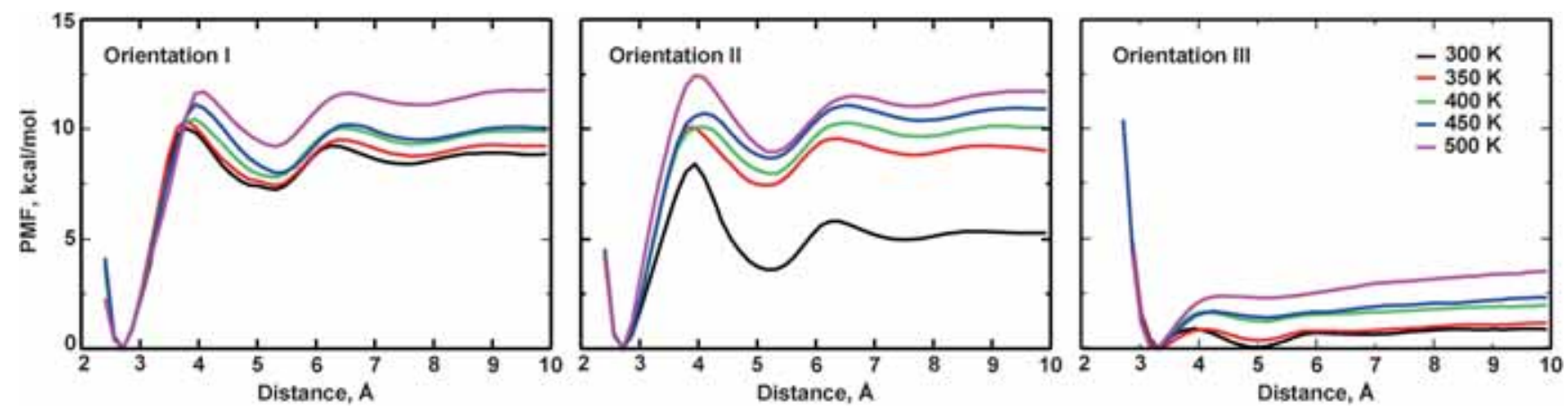

Figure 2. The potential of mean force corresponding to the association of acetate and methylguanidinium ions as a function of the distance between the two ions.
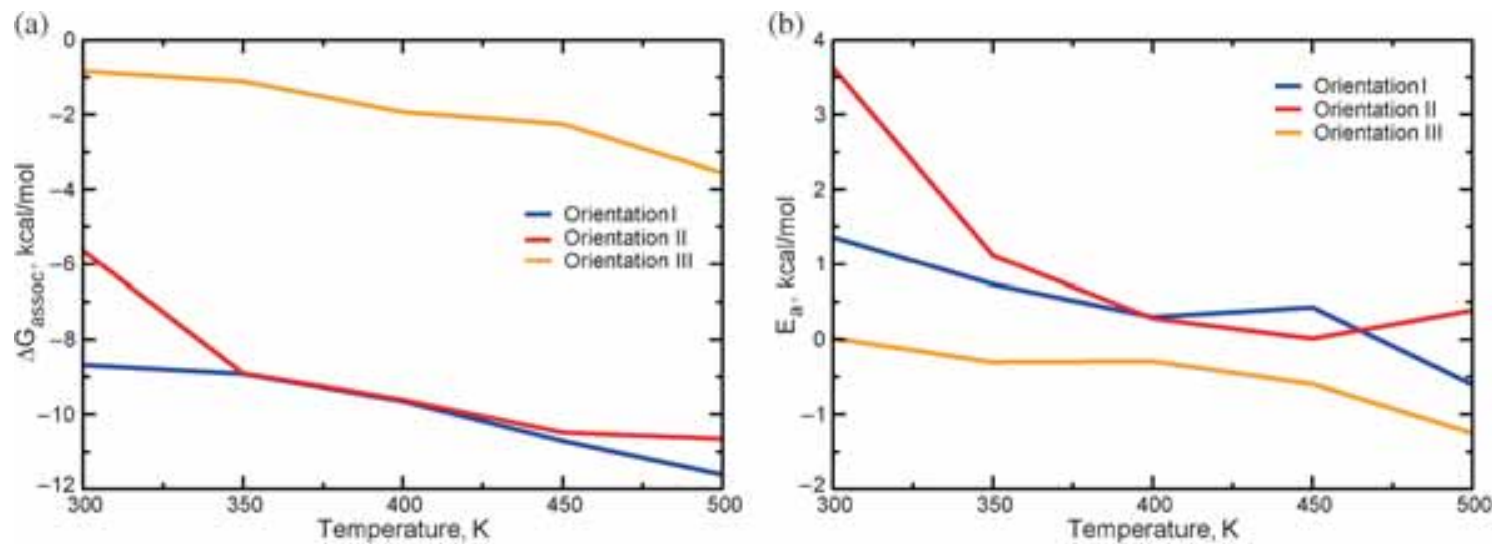

Figure 3. (a) Free energy of association $\Delta G_{a s s o c}$ and (b) activation energy $E_{a}$ corresponding to the association of the ion pair at the different temperatures. 


\section{Results and Discussion}

\subsection{Similarities in the free energy profiles}

for association at different temperatures suggest similar intermediates

The potentials of mean force (PMF) calculated as a function of the distance between acetate and methylguanidium with respect to the three orientations are shown in Figure 2. The free energy landscape is flat beyond a distance of $6.5 \AA$, suggesting that interactions between acetate and methylguanidium beyond this point are not significant. There are two distinct minima, one occurring at a distance of $\sim 2.8 \AA$, and the other at a distance of $\sim 5.3 \AA$. The presence of a deep minimum at $\sim 2.8 \AA$ can be ascribed to strong electrostatic interaction between the two ions. The minimum at farther separation is likely to correspond to a solvent-separated state, as described later. While the free energy profiles are similar for orientations I and II, the free energy profile for orientation III is flat and completely different from the other two orientations. A possible reason for this is the fact that, while the former two orientations involve the interaction of two nitrogen centers on the methylguanidium with the acetate, orientation III involves only one nitrogen center. Furthermore, the depth of the contact minimum in orientation III is the lowest among all three orientations. Although the contact minimum and the solvent separated minimum in orientation III are very similar in energy at $300 \mathrm{~K}$, the solvent separated minimum is destabilized at higher temperatures. Another noteworthy feature is the free energy profile for orientation II at $300 \mathrm{~K}$. The relatively low stabilization of the contact minimum relative to the dissociated state is rather unexpected, and the reason for this is unclear. The trends seen here for the free energy profiles, notably the occurrence of two distinct minima, are consistent with those reported previously for similar systems. ${ }^{28,29,35}$

A noteworthy point to be discussed here is the possible variation of the free energy profile with respect to the force field used. Results obtained for ion pair association, however, are seen to be similar for different force fields, and the results reported in the current study are similar to those reported in previous studies that employed different force fields. ${ }^{26,28,29,32,35} \mathrm{~A}$ set of force field parameters developed recently for acetate and other anions that form ionic liquids with imidazolium based cations have been able to reproduce the physicochemical properties of these ionic liquids fairly accurately. ${ }^{46-48}$ Future studies could employ these force field parameters for acetate in order to examine if there is any variation in the free energy profile, and whether it is different from that obtained using the commonly used force fields.

\subsection{Stabilization due to ion pairs is more pronounced at high temperatures, with relative orientation of ion pairs being crucial}

In order to quantify the stabilization arising from the association of the ions, the overall free energy change corresponding to the association process, $\Delta G_{a s s o c}$, and the activation energy for the association, $E_{a}$, were
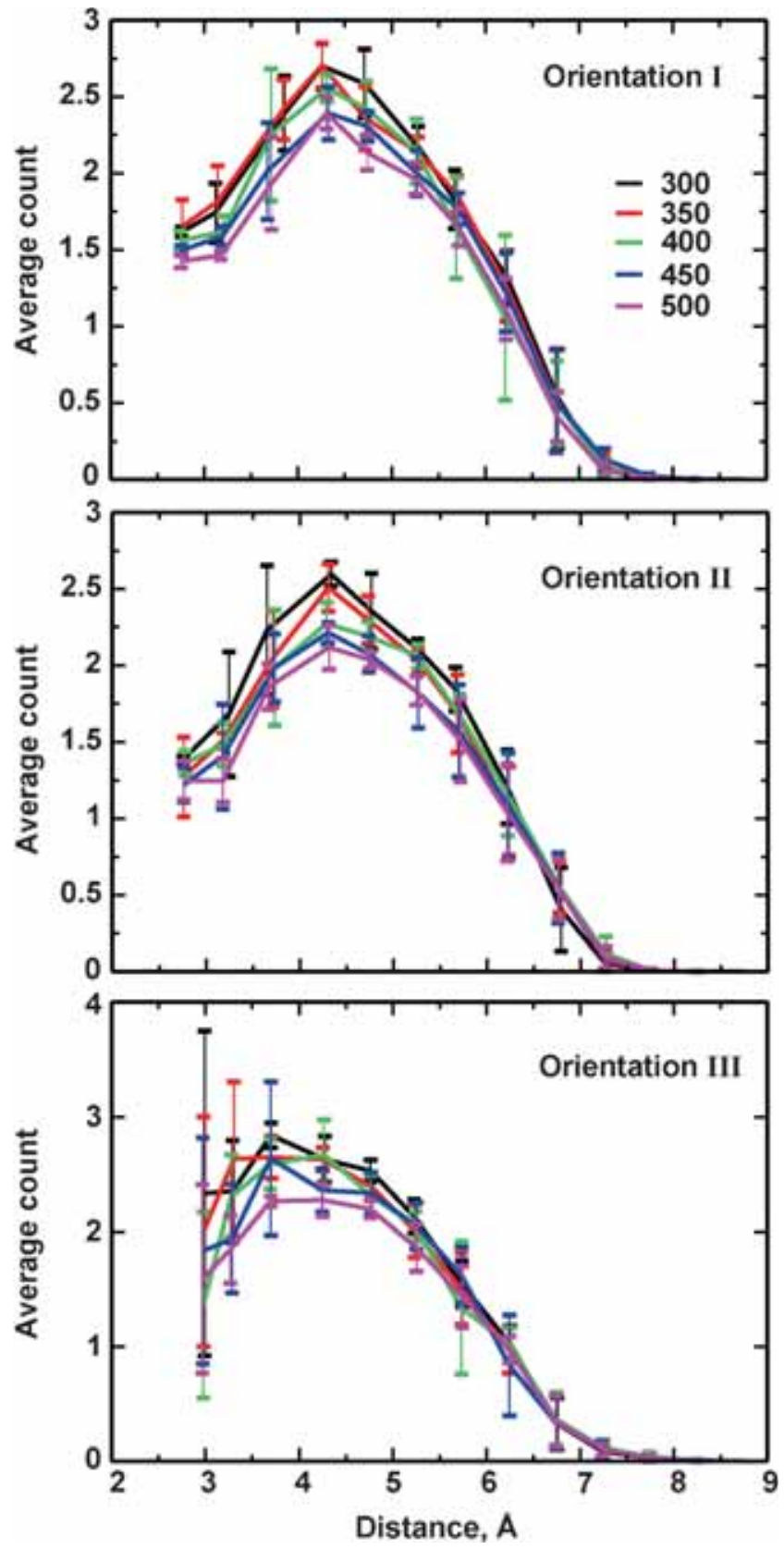

Figure 4. Average number of bridging water molecules between the ions at different temperatures for all the three orientations. 
calculated. The free energy difference between the fully dissociated state of the ions and the contact minimum gave $\Delta G_{a s s o c}$, and the free energy difference between the fully dissociated state and the transition state (occurring between the two energy minima) gave $E_{a}$. Figure 3 shows the change in $\Delta G_{a s s o c}$ and $E_{a}$ with respect to temperature for the three orientations. The magnitude of $\Delta G_{\text {assoc }}$ increases with an increase in temperature for all the three orientations. The stabilization due to the association of ion pairs is thus more pronounced at high temperatures. This also implies that the effect of ion pairs is more favorable at the temperatures at which thermophiles thrive. Thermophiles could possibly use this as a mechanism for preserving the structural integrity of their proteins at high temperatures. This accounts for the high occurrence of ion pairs in thermophilic proteins compared to mesophilic proteins, and could explain the thermostability of the former. ${ }^{49}$ The magnitude of $\Delta G_{a s s o c}$ in orientation III is less than the other two orientations, which is because this orientation involves interaction of one nitrogen center with the acetate, as opposed to two nitrogen centers in the other two orientations.

The activation energies in Figure 3 show that the energy barrier for association decreases with an increase in temperature. Thus, the rate of association of ion pairs is faster at higher temperatures compared to physiological temperatures. It is to be noted that, in terms of both $\Delta G_{a s s o c}$ and $E_{a}$, orientation I is more favorable than the other two orientations, and this is likely to be the mode via which ion pairs in thermophilic proteins actually interact. This orientation differs from the other two interactions in that the interaction between the oppositely charged groups is "head-to-head" rather than "head-to-side" or "side-toside". This is in agreement with previous free energy calculations on salt bridges, which proposed that interactions between side chains in a salt bridge are strongest when the side chains are coaxial. ${ }^{26}$ It follows that salt bridges will be strongest when there is head-to-head interaction between the side chains. To sum up the findings based on $\Delta G_{a s s o c}$ and $E_{a}$, it is seen that $\Delta G_{a s s o c}$ becomes more negative with an increase in temperature, while the energy barrier becomes lower. Thus, high temperature makes the association of ion pairs favorable both thermodynamically and kinetically.

\subsection{Bridging water molecules between ion pairs suggest a role for internal water in thermostable proteins}

The free energy profile shows a second minimum around $5.3 \AA$ (Figure 2), which corresponds to a solvent-separated state. To investigate the nature of such a state, the number of bridging water molecules between the ion pairs was calculated (Figure 4). The maximum number of bridging water molecules between the two ions corresponds to the second energy minimum in the PMF. At such separations, the ions are too far away to be able to form direct interactions, and the existence of bridging water molecules is necessary to hold together the ions. It is also interesting to note that, even in the state corresponding to the contact minimum, there are one or two bridging water molecules. The presence of bridging water molecules offers an explanation for the existence of internal water
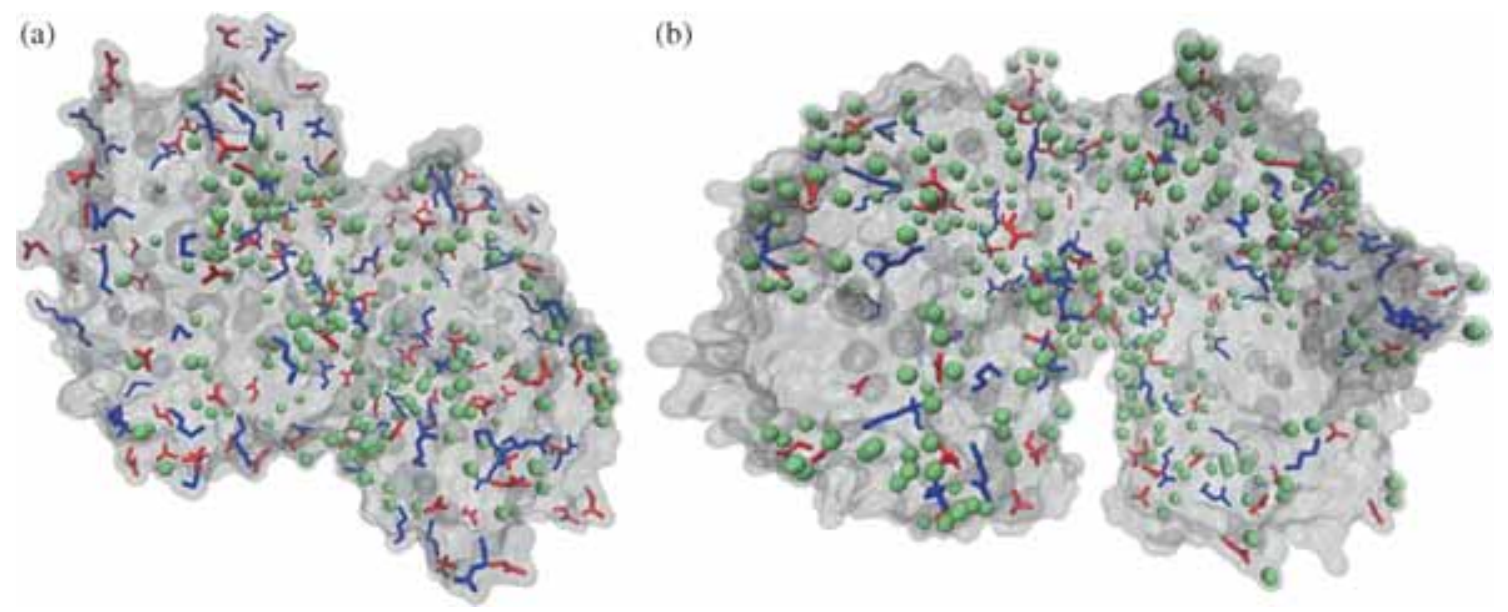

Figure 5. Crystal structures of two thermophilic proteins with internal water molecules. Positively charged residue side chains are shown in blue, negatively charged side chains in red, and water oxygen atoms in green. Some water molecules on the surface of the protein have been omitted for clarity. (a) Malate dehydrogenase single site mutant T198I from Thermus thermophilus with PDB ID 1BDM. (b) Malate dehydrogenase from Chloroflexus aurantiacus with PDB ID 4CL3. 
molecules in thermostable proteins. ${ }^{24,25,50,51}$ In general, there are fewer water molecules in between the two ions at higher temperatures. This is analogous to the dehydration of internal cavities in thermophilic proteins at high temperatures, with interactions between ion pairs being more direct rather than being bridged via water molecules. ${ }^{24}$

An illustration of the occurrence of internal water molecules in thermophilic proteins is shown in Figure 5. The figure shows experimental structures of two thermostable proteins along with water molecules reported in the crystal structure. ${ }^{50,51}$ The structures of the two proteins show that, in addition to a few salt bridges that involve direct interactions between oppositely charged residues, there are a number of salt bridges with water molecules occurring in between two oppositely charged
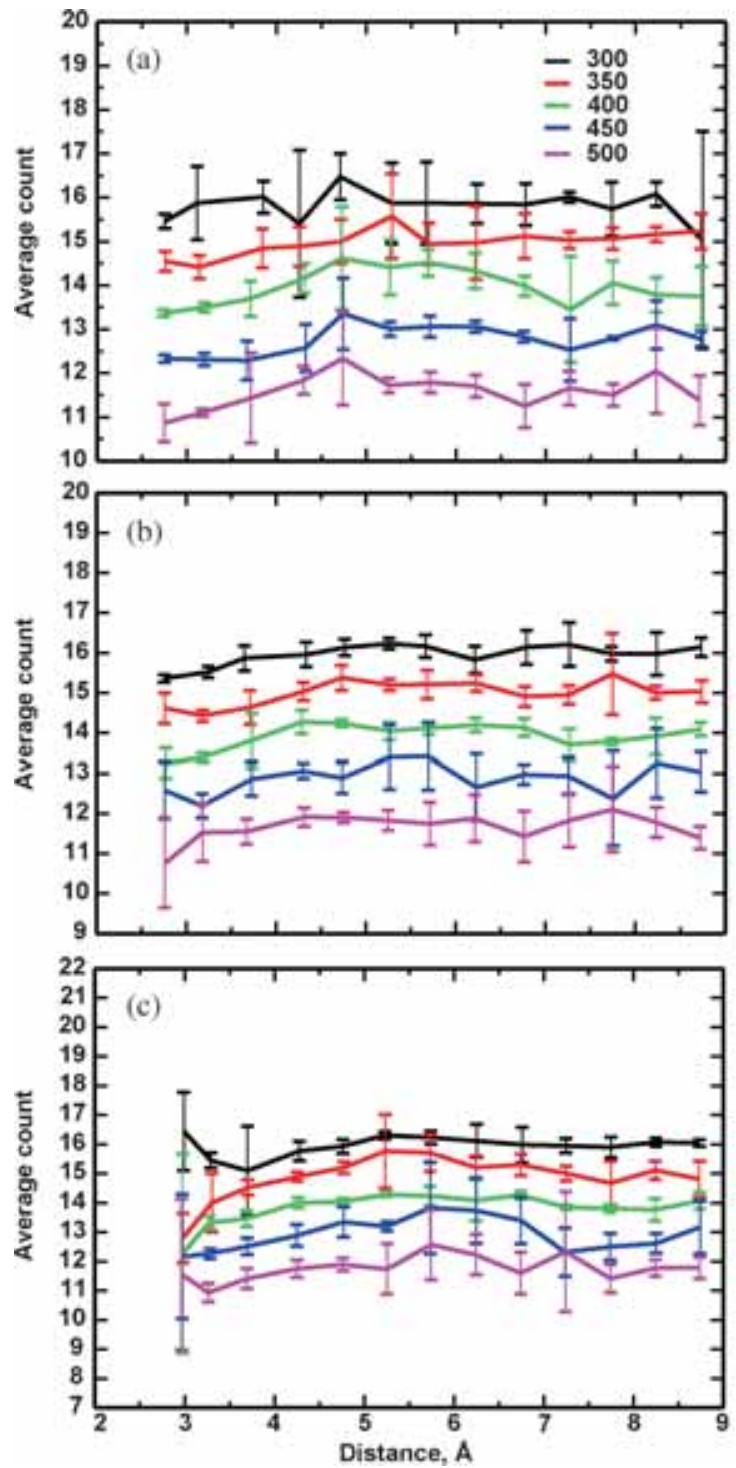

residues. Although internal water molecules have not been reported as a structural feature that is necessary for thermostability, the fact that they do occur in a number of thermophilic proteins implies that their importance in the thermostability of these proteins cannot be ignored. ${ }^{24,25,50,51}$

\subsection{Optimal ion pair interactions are ensured via compensation between desolvation and electrostatic interactions}

In order to quantify the effect of solvation on the ion pair, the hydration number and solvation energy of the ions were calculated, and are shown in Figures 6 and 7 , respectively. The hydration number for a given ion was calculated by finding the number of water oxygen
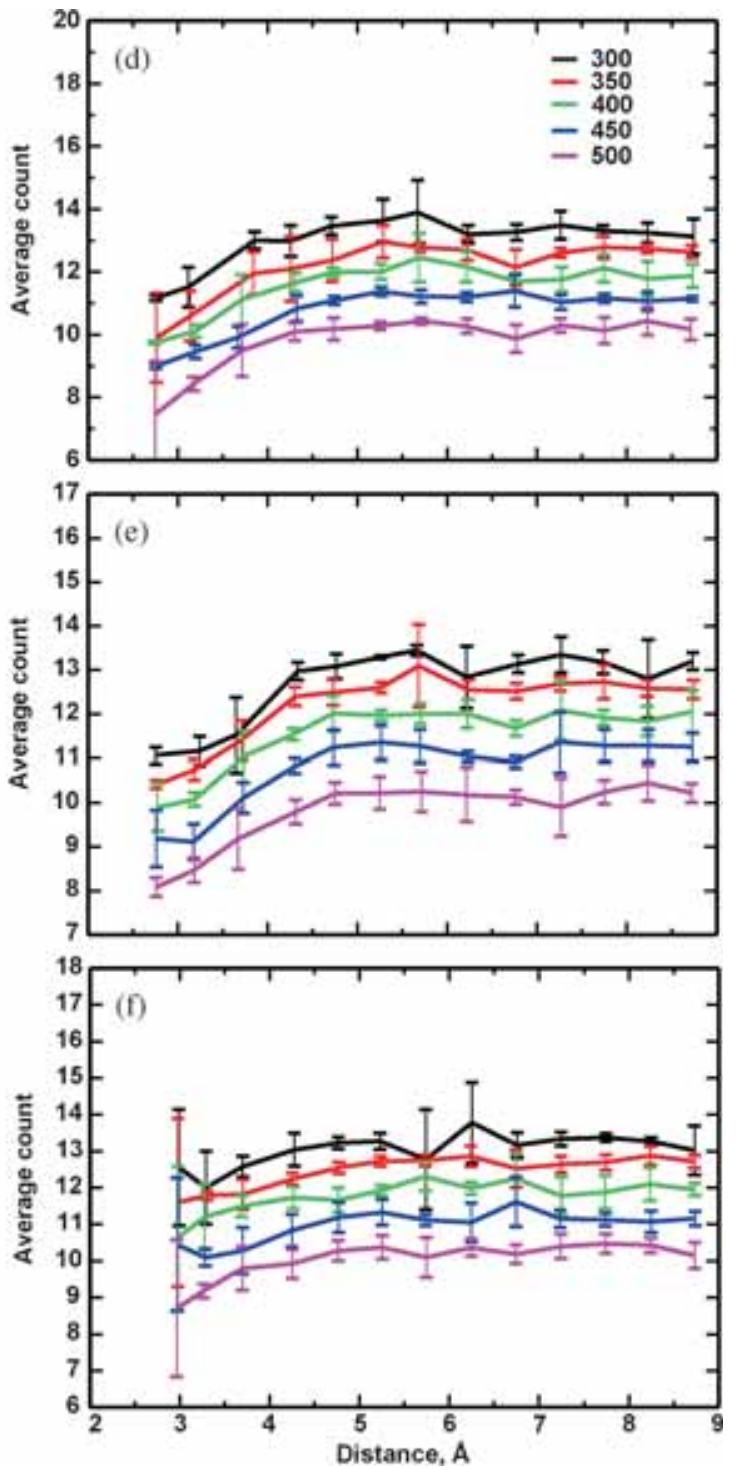

Figure 6. Hydration number of methylguanidium (a, b, c) and acetate (d, e, f) ions. The hydration number was determined by calculating the number of oxygen atoms within $3.5 \AA$ of the ions. The values shown have been averaged over bins along the reaction coordinate. 

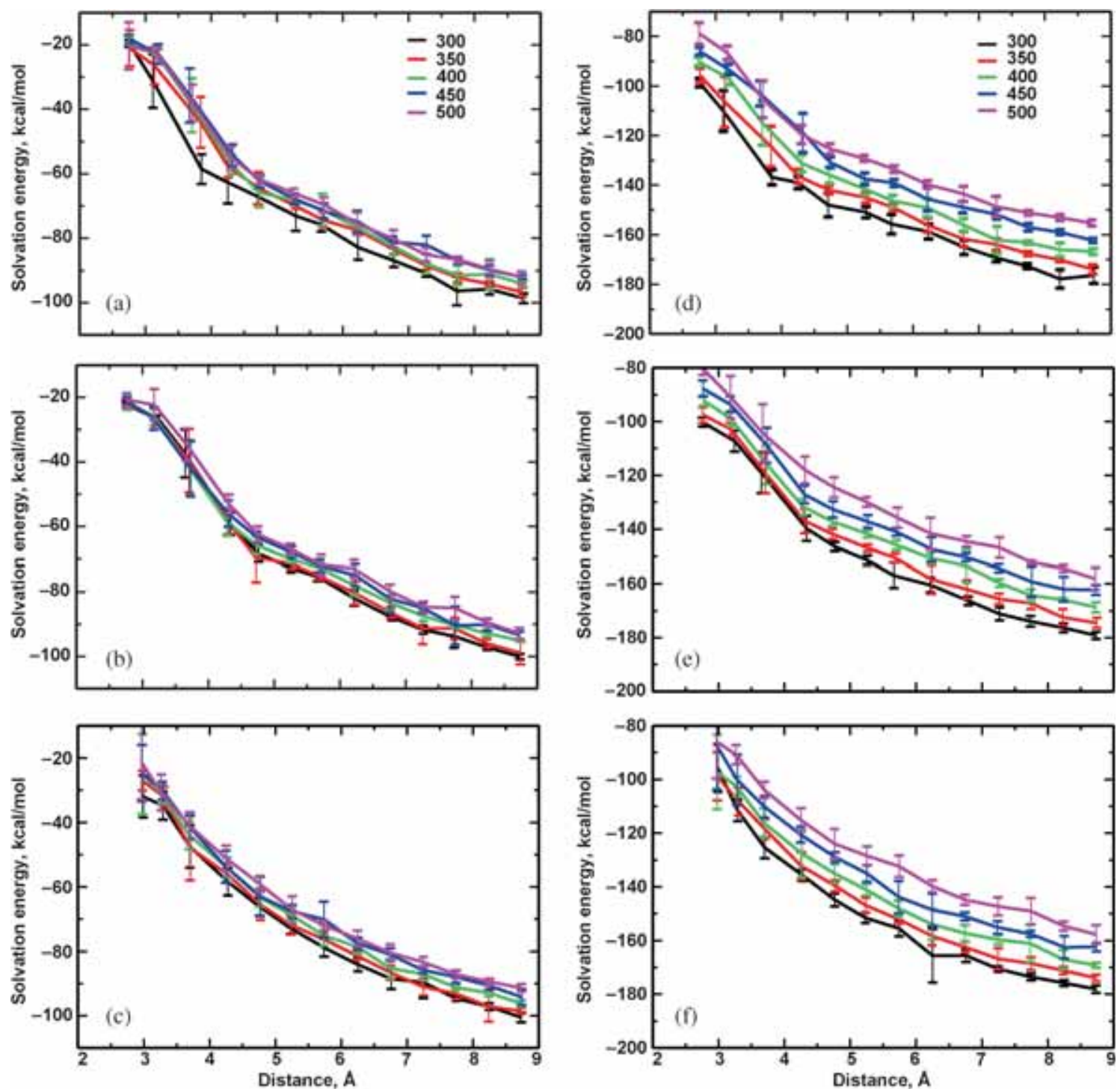

Figure 7. Solvation energy of methylguanidium (a, b, c) and acetate (d, e, f) ions in orientations I, II and III. The values shown have been averaged over bins along the reaction coordinate.

atoms within a radius of $3.5 \AA$ of the ion. The ions are seen to possess fewer coordinating water molecules in the associated form compared to the fully separated form (Figure 6). Furthermore, the hydration number decreases with an increase in temperature, which is because the higher kinetic energy possessed by water molecules at these temperatures helps them overcome stable interactions with the ions, and consequently, there are fewer number of water molecules interacting with these ions. Furthermore, there is a decreased density of water molecules at high temperatures, owing to a slight expansion of the box. At high temperatures, the ions are not fully solvated even in the fully separated state, and it follows that the loss in coordinating water molecules associated with ion association at high temperatures is less drastic compared to that at lower temperatures. The solvation energies for the two ions were analyzed to further support this (Figure 7). For methylguanidium, the solvation energies clearly indicate that the desolvation penalty accompanying ion association is lower at high temperature. However, for acetate, the desolvation penalties for low and high temperatures are almost the same. Thus, the total solvation energy for both ions taken together, and the total hydration number, were calculated to estimate the overall effect of solvation on the ions (Figure 8). The values clearly indicate that the overall desolvation penalty is lower at high temperatures.

The most favorable state for the ion pair occurs at a separation of $\sim 2.8 \AA$ (Figure 2). This state involves very strong electrostatic interactions between the ions, with one or two bridging water molecules. While the close separation ensures that there are strong electrostatic interactions, the bridging water molecules ensure 

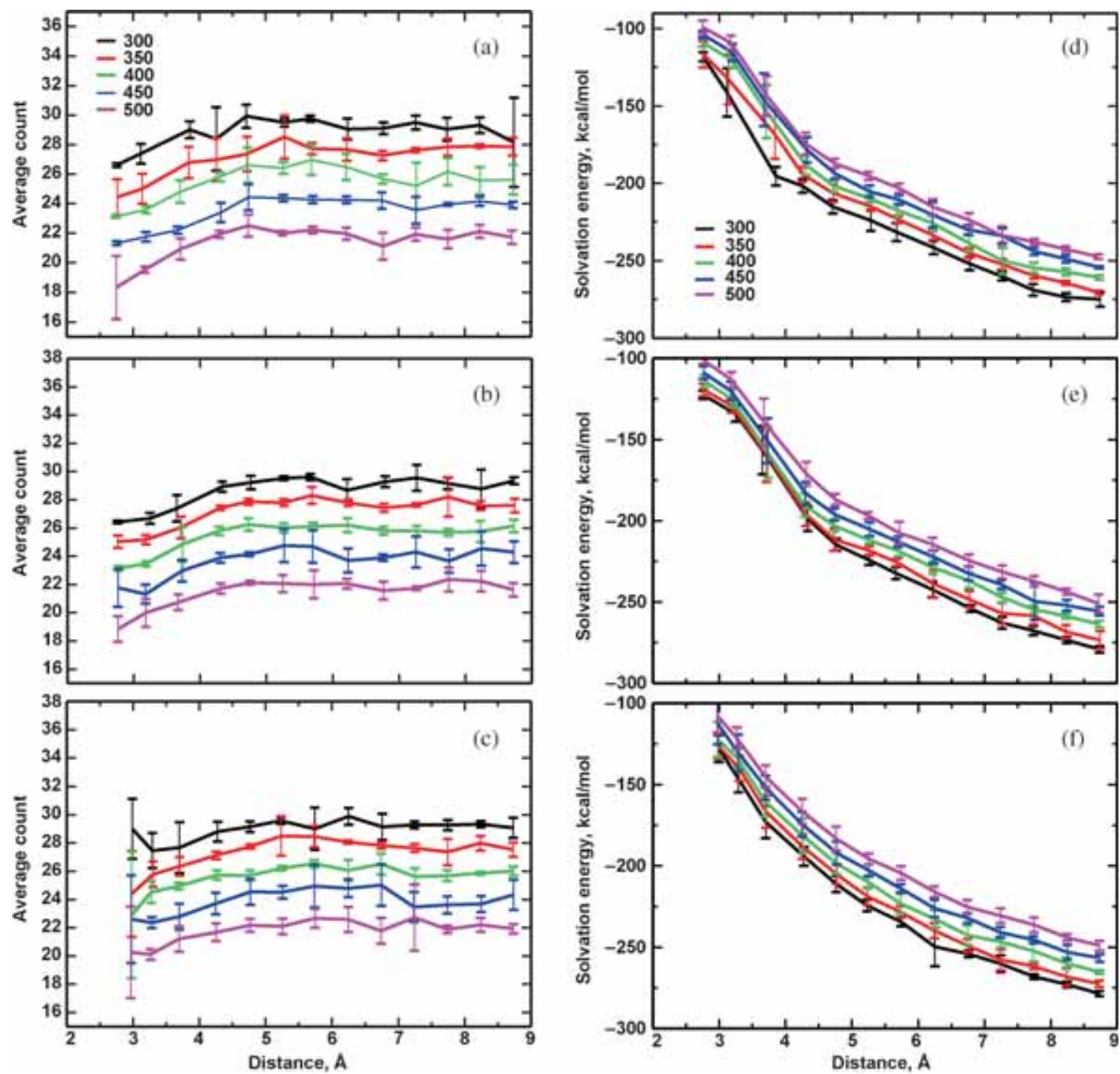

Figure 8. (a, b, c) Overall hydration number of the two ions in orientations I, II and III and (d, e, f) the sum of the individual solvation energies of the two ions in orientations I, II and III.

that the ions are not completely desolvated. Thus, there is compensation between direct ion-ion interactions and ion solvation. The desolvation penalty corresponding to ion association is the lowest at high temperatures, and it follows that the formation of ion pairs is more favorable at high rather than low temperatures. This explains why thermophilic proteins possess greater number of ion pairs compared to mesophilic proteins. ${ }^{49}$

\section{Conclusions}

The association of an ion pair has been studied in atomistic detail in order to investigate the role of temperature and ion solvation. Free energy profiles suggest that the most favorable state occurs at a separation of $\sim 2.8 \AA$, with one or two bridging water molecules between the ions. This offers an explanation for the existence of internal water molecules in thermostable proteins proposed recently. ${ }^{23,24,50,51}$ The mode of interaction of the ion pair is via head-to-head interaction of the oppositely charged ion pair, and the association of the ion pair is a balance between direct ion-ion interactions and the extent of solvation of the ions. Furthermore, the desolvation penalty associated with the formation of the ion pairs decreases with an increase in temperature, and, consequently, the stabilization due to the association of the ions is more significant at higher temperatures. On the whole, the results show that the prevalence of ion pairs is a crucial determining factor of thermostability, explaining why they are more conserved in thermophilic rather than mesophilic proteins. Further studies on other model systems such as ammonium and imidazolium ions using different force fields and water models are proposed for further understanding of this phenomenon. 


\section{Acknowledgments}

This work was supported by the Department of Science and Technology, Government of India under the Women Scientists Scheme (grant no. SR/WOS-A/LS-1272/2014(G)).

\section{References}

1. Stetter K O 2006 Hyperthermophiles in the history of lifePhil Trans. R. Soc. B 3611837

2. Unsworth L D, Oost J V D and Koutsopoulos S 2007 Hyperthermophilic enzymes-stability, activity and implementation strategies for high temperature applications FEBS J. 2744044

3. Kashefi K 2003 Extending the upper temperature limit for life Science 301934

4. Luke K A, Higgins C L and Wittung-Stafshede P 2007 Thermodynamic stability and folding of proteins from hyperthermophilic organisms FEBS J. 2744023

5. Vieille C and Zeikus G J 2001 Hyperthermophilic enzymes: Sources, uses, and molecular mechanisms for thermostability Microbiol. Mol. Biol. Rev. 651

6. Singh B, Bulusu G and Mitra A 2015 Understanding the thermostability and activity of Bacillus subtilis lipase mutants: Insights from molecular dynamics simulations J. Phys. Chem. B 119392

7. Lee C-W, Wang H-J, Hwang J-K and Tseng C-P 2014 Protein thermal stability enhancement by designing salt bridges: A combined computational and experimental study PLoS ONE 9 e 112751

8. Porebski B T, Nickson A A, Hoke D E, Hunter M R, Zhu L, McGowan S, Webb G I and Buckle A M 2015 Structural and dynamic properties that govern the stability of an engineered fibronectin type III domain Protein Eng. Des. Sel. 2867

9. Bouzas T D M, Barros-Velazquez J and Villa T G 2006 Industrial applications of hyperthermophilic enzymes: A review Protein Pept. Lett. 13645

10. Blumer-Schuette S E, Kataeva I, Westpheling J, Adams M W and Kelly R M 2008 Extremely thermophilic microorganisms for biomass conversion: Status and prospects Curr. Opin. Biotechnol. 19210

11. Choi J-M, Han S-S and Kim H-S 2015 Industrial applications of enzyme biocatalysis: Current status and future aspects Biotechnol. Adv. $\mathbf{3 3} 1443$

12. Rathi P C, Höffken H W and Gohlke H 2014 Quality matters: Extension of clusters of residues with good hydrophobic contacts stabilize (hyper)thermophilic proteins J. Chem. Inf. Model. 54355

13. Gromiha M M, Pathak M C, Saraboji K, Ortlund E A and Gaucher E A 2013 Hydrophobic environment is a key factor for the stability of thermophilic proteins Proteins $\mathbf{8 1} 715$

14. Kumar S, Tsai C-J and Nussinov R 2000 Factors enhancing protein thermostability Protein Eng. Des. Sel. 13179

15. Yip K, Stillman T, Britton K, Artymiuk P, Baker $\mathrm{P}$, Sedelnikova S, Engel P, Pasquo A, Chiaraluce R, Consalvi V, Scandurra R and Rice D 1995 The structure of Pyrococcus furiosus glutamate dehydrogenase reveals a key role for ion-pair networks in maintaining enzyme stability at extreme temperatures Structure 31147

16. Missimer J H, Steinmetz M O, Baron R, Winkler F K, Kammerer R A, Daura X and Gunsteren W F V 2007 Configurational entropy elucidates the role of saltbridge networks in protein thermostability Protein Sci. 161349

17. Priyakumar U D 2012 Role of hydrophobic core on the thermal stability of proteins - molecular dynamics simulations on a single point mutant of Sso7d J. Biomol. Struct. Dyn. 29961

18. Priyakumar U D, Ramakrishna S, Nagarjuna K R and Reddy S K 2010 Structural and energetic determinants of thermal stability and hierarchical unfolding pathways of hyperthermophilic proteins, Sac7d and Sso7d J. Phys. Chem. B 1141707

19. Priyakumar U D, Harika G and Suresh G 2010 Molecular simulations on the thermal stabilization of DNA by hyperthermophilic chromatin protein Sac7d, and associated conformational transitions J. Phys. Chem. B 114 16548

20. Manjunath K and Sekar K 2013 Molecular dynamics perspective on the protein thermal stability: A case study using SAICAR synthetase J. Chem. Inf. Model. 532448

21. Merkley E D, Parson W W and Daggett V 2010 Temperature dependence of the flexibility of thermophilic and mesophilic flavoenzymes of the nitroreductase fold Protein Eng. Des. Sel. 23327

22. Mamonova T B, Glyakina A V, Kurnikova M G and Galzitskaya O V 2010 Flexibility and mobility in mesophilic and thermophilic homologous proteins from molecular dynamics and FoldUnfold method J. Bioinform. Computat. Biol. 08377

23. Rahaman O, Kalimeri M, Melchionna S, Hénin J and Sterpone F 2015 Role of internal water on protein thermal stability: The case of homologous $\mathrm{G}$ domains $J$. Phys. Chem. B 1198939

24. Chakraborty D, Taly A and Sterpone F 2015 Stay wet, stay stable? How internal water helps the stability of thermophilic proteins J. Phys. Chem. B 11912760

25. Sterpone F, Bertonati $C$, Briganti $G$ and Melchionna S 2009 Key role of proximal water in regulating thermostable proteins J. Phys. Chem. B 113131

26. Masunov A and Lazaridis T 2003 Potentials of mean force between ionizable amino acid side chains in water J. Am. Chem. Soc. 1251722

27. Kumar S and Nussinov R 2002 Relationship between ion pair geometries and electrostatic strengths in proteins Biophys. J. 831595

28. Thomas A S and Elcock A H 2004 Molecular simulations suggest protein salt bridges are uniquely suited to life at high temperatures J. Am. Chem. Soc. 1262208

29. Elcock A H 1998 The stability of salt bridges at high temperatures: Implications for hyperthermophilic proteins J. Mol. Biol. 284489

30. Yang H and Elcock A H 2003 Association lifetimes of hydrophobic amino acid pairs measured directly from molecular dynamics simulations J. Am. Chem. Soc. 125 13968

31. Iwahara J, Esadze A and Zandarashvili L 2015 Physicochemical properties of ion pairs of biological macromolecules Biomolecules 52435 
32. Thomas A S and Elcock A H 2006 Direct observation of salt effects on molecular interactions through explicit-solvent molecular dynamics simulations: Differential effects on electrostatic and hydrophobic interactions and comparisons to Poisson-Boltzmann theory J. Am. Chem. Soc. 1287796

33. Thomas A S and Elcock A H 2007 Molecular dynamics simulations of hydrophobic associations in aqueous salt solutions indicate a connection between water hydrogen bonding and the Hofmeister effect J. Am. Chem. Soc. 12914887

34. Thomas A S and Elcock A H 2011 Direct measurement of the kinetics and thermodynamics of association of hydrophobic molecules from molecular dynamics simulations J. Phys. Chem. Lett. 219

35. Zhu S and Elcock A H 2010 A complete thermodynamic characterization of electrostatic and hydrophobic associations in the temperature range 0 to $100^{\circ} \mathrm{C}$ from explicit-solvent molecular dynamics simulations $J$. Chem. Theory Comput. 61293

36. Debiec K T, Gronenborn A M and Chong L T 2014 Evaluating the strength of salt bridges: A comparison of current biomolecular force fields J. Phys. Chem. B 118 6561

37. Brooks B R, Brooks C L, MacKerell A D, Nilsson L, Petrella R J, Roux B, Won Y, Archontis G, Bartels C, Boresch S, Caflisch A, Caves L, Cui Q, Dinner A R, Feig M, Fischer S, Gao J, Hodoscek M, Im W, Kuczera K, Lazaridis T, Ma J, Ovchinnikov V, Paci E, Pastor R W, Post C B, Pu J Z, Schaefer M, Tidor B, Venable R M, Woodcock H L, Wu X, Yang W, York D M and Karplus M 2009 CHARMM: The biomolecular simulation program J. Comput. Chem. 301545

38. MacKerell A D, Feig M and Brooks C L 2004 Extending the treatment of backbone energetics in protein force fields: Limitations of gas-phase quantum mechanics in reproducing protein conformational distributions in molecular dynamics simulations J. Comput. Chem. 25 1400

39. MacKerell A D, Bashford D, Bellott M, Dunbrack R L, Evanseck J D, Field M J, Fischer S, Gao J, Guo H, Ha S, Joseph-McCarthy D, Kuchnir L, Kuczera K, Lau F T K, Mattos C, Michnick S, Ngo T, Nguyen D T, Prodhom B, Reiher III W E, Roux B, Schlenkrich M, Smith J C, Stote T, Straub J E, Watanabe M, Wiorkiewicz-Kuczera J, Yin D and Karplus M 1998 All-atom empirical potential for molecular modeling and dynamics studies of proteins $J$. Phys. Chem. B 1023586

40. Jorgensen W L, Chandrasekhar J, Madura J D, Impey R W and Klein M L 1983 Comparison of simple potential functions for simulating liquid water J. Chem. Phys. 79926

41. Darden T, York D and Pedersen L 1993 Particle mesh Ewald: An $N \cdot \log (N)$ method for Ewald sums in large systems J. Chem. Phys. 9810089

42. Ryckaert J-P, Ciccotti G and Berendsen H J 1977 Numerical integration of the cartesian equations of motion of a system with constraints: Molecular dynamics of n-alkanes J. Comput. Phys. 23327

43. Kumar S, Rosenberg J M, Bouzida D, Swendsen R H and Kollman P A 1992 The weighted histogram analysis method for free-energy calculations on biomolecules. I. The method J. Comput. Chem. 131011

44. Grossfield A "WHAM: the weighted histogram analysis method", http://membrane.urmc.rochester.edu/content/ wham Date of access: 17.01.2017

45. Humphrey W, Dalke A and Schulten K 1996 VMD: Visual Molecular Dynamics J. Mol. Graphics 1433

46. Mondal A and Balasubramanian S 2015 A refined allatom potential for imidazolium-based room temperature ionic liquids: Acetate, dicyanamide, and thiocyanate anions J. Phys. Chem. B 11911041

47. Mondal A and Balasubramanian S 2014 Quantitative prediction of physical properties of imidazolium based room temperature ionic liquids through determination of condensed phase site charges: A refined force field $J$. Phys. Chem. B 1183409

48. Mondal A and Balasubramanian S 2014 A molecular dynamics study of collective transport properties of imidazolium-based room-temperature ionic liquids $J$. Chem. Eng. Data 593061

49. Szilágyi A and Závodszky P 2000 Structural differences between mesophilic, moderately thermophilic and extremely thermophilic protein subunits: Results of a comprehensive survey Structure 8493

50. Kelly C A, Nishiyama M, Ohnishi Y, Beppu T and Birktoft J J 1993 Determinants of protein thermostability observed in the 1.9-A crystal structure of malate dehydrogenase from the thermophilic bacterium Thermus flavus Biochemistry 323913

51. Talon R, Coquelle N, Madern D and Girard E 2014 An experimental point of view on hydration/solvation in halophilic proteins Front. Microbiol. 566 